\title{
A Unified Approach to Convergence Analysis of Discretization Methods for Volterra-type Equations
}

\author{
Jennifer Dixon and Sean McKee \\ Oxford University Computing Laboratory. \\ 8-11 Keble Road, Oxford
}

[Received 27 July 1983 and in revised form 28 March 1984]

\begin{abstract}
This paper presents a general convergence analysis of numerical methods for solving ordinary differential equations and non-linear Volterra integral and integrodifferential equations. The concept of analytic and discrete fundamental forms is introduced. Prolongation and restriction operators reduce the problem of comparing the analytic and numerical solutions to that of considering the effect of perturbations in the fundamental forms. Integral inequalities and their discrete analogues are then employed to derive error estimates. The theory is illustrated by a convergence proof of a collocation method for solving Volterra integral equations of the second kind.
\end{abstract}

\section{Introduction}

WE SHALL BE CONCERNED with the numerical solution of a non-linear operator equation

$$
\Phi(y)=0 \text {, }
$$

where $\Phi: X \rightarrow Y$ is a non-linear operator from some normed linear space $X$ into a linear space $Y$. It will be assumed throughout that the true solution $y \in X$ of the original problem (1.1) exists uniquely.

To approximate (1.1) we introduce the discretization

$$
\Phi^{h}\left(y^{h}\right)=0,
$$

where for each $h$ belonging to some non-empty set $I \subset \mathbf{R}^{+}$with inf $I=0$, $\Phi^{k}: X^{k} \rightarrow Y^{k}$ is a non-linear operator from a normed linear space $X^{k}$ into a linear space $Y^{k}$.

The basic idea of a discretization method is to replace the original problem by one depending on a parameter $h$ and to consider an infinite sequence of such replacements corresponding to a sequence of values of $h$ with limit zero. The replacement is to be such that the further one proceeds in the sequence the more accurate the approximations (in a sense to be defined) given by the solution of the discretization are to the solution of the original problem.

In general the solution $y \in X$ of the original problem and the solution $y^{h} \in X^{h}$, $h \in I$, of its discretization belong to different spaces and cannot immediately be related. There are essentially two ways to effect a comparison: 
(i) Define with the discretization method a sequence of prolongation operators $p^{h}: X^{h} \rightarrow X$ and consider the closeness of $p^{h} y^{h}$ and $y$ in $X$. The sequence $\left\{p^{k} y^{k}\right\}_{1, I}$ will be said to $p$-converge to $y \in X$ if $\left\|p^{k} y^{k}-y\right\|_{X} \rightarrow 0$ as $h \rightarrow 0$.

(ii) Define a sequence of restriction operators $r^{h}: X \rightarrow X^{k}$ and compare $r^{h} y$ and $y^{k}$ in $X^{k}$ for each $h \in I$. The sequence $\left\{y^{k}\right\}_{k}$, will be said to $r$-converge to $y \in X$ if $\left\|r^{k} y-y^{k}\right\|_{x^{h}} \rightarrow 0$ as $h \rightarrow 0$.

Prolongation and restriction operators have been widely used in the error analysis of collocation schemes for solving Fredholm integral equations, for example, see Noble (1973), Thomas (1973) and Wright (1982). Further, they have been applied extensively by the French school in connection with elliptic systems. We refer, for instance, to Aubin (1972) and Céa (1964).

The aim of this paper is to use prolongation and restriction operators to simplify and unify convergence analysis by setting up a general framework which will allow the simultaneous consideration of both approaches for numerical methods for initial-value ordinary differential equations, first- and second-kind Volterra integral equations and Volterra integro-differential equations.

\section{The Analytic Problem}

For simplicity and to illustrate our approach we will initially consider the case $X=C[0, T]$, the Banach space of continuous functions $x$ defined on the interval $[0, T]$ with the supremum norm, $\|x\|_{\infty}=\sup \{|x(t)|: t \in[0, T]\}$. In Sections 5 and 6 the analysis will be extended to more general Banach spaces.

We begin by introducing the concept of a fundamental form.

Definition 2.1. The original problem (1.1) will be said to have an analytic fundamental form (i.e. to be of Volterra type) if it can be expressed in the form

$$
y=g+H(y) \text {, }
$$

where $g \in C[0, T]$ and $H: C[0, T] \rightarrow C[0, T]$ satisfies

$$
|H(y)(t)-H(z)(t)| \leqslant(K|y-z|)(t)
$$

for all $t \in[0, T]$, and all $y, z \in C[0, T]$,

where

$$
K y(t)=\int_{0}^{t} k(t, s) y(s) d s, \quad t \in[0, T],
$$

and the Volterra kernel $k(t, s)$ satisfies the following conditions:

(CI) $k(t, s)$ is non-negative on the triangle $0 \leqslant s \leqslant t \leqslant T$,

(CII) for each $t \in[0, T], k(t, s)$ is integrable over $(0, t)$ as a function of $s$,

(CIII) there exists $\mu \in \mathbf{N}$ such that the $\mu$ th iterated kernel $k^{(\mu)}(t, s)$ of $k(t, s)$ is continuous in $t, s$.

The following theorem is an existence result for the analytic fundamental form. 
THEOREM 2.1 The analytic fundamental form defined by definition 2.1 has a unique solution $y \in C[0, T]$.

Proof. Let $f$ satisfy the Volterra integral equation

$$
f(t)=1+\int_{0}^{t} k(t, s) f(s) d s, \quad 0 \leqslant t \leqslant T,
$$

where $k(t, s)$ is defined in Definition 2.1 .

It can be shown that the conditions (CI)-(CIII) imply (2.3) has a unique solution $f \in C[0, T]$ and that $f(t) \geqslant 1,0 \leqslant t \leqslant T$. Consequently there exists a constant $C<1$ such that

$$
0 \leqslant \frac{1}{f(t)} \int_{0}^{t} k(t, s) f(s) d s=1-\frac{1}{f(t)} \leqslant C .
$$

Define a norm $\|\cdot\|$ on $C[0, T]$ by

$$
\|y\|=\sup \left\{\frac{1}{f(t)}|y(t)|: t \in[0, T]\right\}
$$

where $f$ is the solution of (2.3).

Let $F: C[0, T] \rightarrow C[0, T]$ be the non-linear operator defined by

Using (2.2)

$$
F(y)=g+H(y) \text {. }
$$

$$
|F(y)(t)-F(z)(t)| \leqslant \int_{0}^{t} k(t, s)|y(s)-z(s)| d s
$$

and with the aid of (2.4) it follows that

$$
\begin{aligned}
\frac{1}{f(t)}|F(y)(t)-F(z)(t)| & \leqslant \frac{1}{f(t)} \int_{0}^{t} k(t, s) f(s) d s\|y-z\| \\
& \leqslant C\|y-z\| .
\end{aligned}
$$

This holds for all $t \in[0, T]$ and hence taking the supremum

$$
\|F(y)-F(z)\| \leqslant C\|y-z\| \text {. }
$$

Thus since $C<1, F$ is a contraction mapping and by the Banach Fixed Point Theorem we deduce that there exists a unique $y \in C[0, T]$ such that

that is,

$$
F(y)=y \text {, }
$$

$$
y=g+H(y) \text {. }
$$

The analytic fundamental form is convenient for considering the effect of perturbations and the stability of the solution $y$ of the original problem (1.1) since the following linear generalization of Gronwall's inequality given by Dixon and McKee (1984) is applicable.

THEOREM 2.2 For any $\phi \in C[0, T]$ if $x \in C[0, T]$ satisfies the integral inequality

$$
x(t) \leqslant \phi(t)+\int_{0}^{t} k(t, s) x(s) d s, \quad 0 \leqslant t \leqslant T
$$


where the Volterra kernel satisfies conditions $(\mathrm{CI})-(\mathrm{CIII})$, then

$$
x(t) \leqslant y(t), \quad 0 \leqslant t \leqslant T,
$$

where $y$ is the unique $C[0, T]$ solution of the integral equation

$$
y(t)=\phi(t)+\int_{0}^{t} k(t, s) y(s) d s, \quad 0 \leqslant t \leqslant T .
$$

Moreover, there exists a constant $C$ independent of $\phi$ such that if $x \geqslant 0$ and $\phi \geqslant 0$

$$
\|x\|_{\infty} \leqslant C\|\phi\|_{\infty} .
$$

To illustrate the reduction of the original problem to analytic fundamental form consider the ordinary differential equation

$$
\frac{d y}{d t}(t)=f(t, y(t)), \quad y(0)=y_{0}, \quad 0 \leqslant t \leqslant T .
$$

Integrating over $(0, t)$ yields

$$
y(t)=y_{0}+\int_{0}^{t} f(s, y(s)) d s, \quad 0 \leqslant t \leqslant T,
$$

which is clearly the analytic fundamental form for (29) with the kernel $k(t, s)=L$, $0 \leqslant s \leqslant t \leqslant T$, where $L$ is the Lipschitz constant of $f(t, y)$ with respect to $y$.

\section{The Discrete Problem}

As the parameter $h$ tends to zero we may anticipate that the discrete operator will in some sense tend to that of the original equation and, therefore, it seems natural to expect that any analysis for the continuous problem will have a parallel in the approximating problem.

Following this view we introduce a discrete version of the space $C[0, T]$, replacing continuous functions by sequences of finite real numbers. It is assumed that the true solution is to be approximated on some finite interval $[0, T]$.

Definition 3.1. Let $T$ and $h_{0}$ be given with $h_{0}$ such that $T / h_{0}=N_{0}$, a positive integer, and let $I$ be the set defined by

$$
I:=\left\{h: h=T / N, N \in \mathbf{N}, N \geqslant N_{0}\right\} .
$$

For each $h \in I$ let $\Omega^{h}=\{0,1, \ldots, N\}$. Define

$$
C\left(\Omega^{h}\right):=\left\{y^{k}=\left(y_{i}\right): i \in \Omega^{h}, y_{i} \in \mathbb{R},\left\|y^{h}\right\|_{\infty}=\max \left\{\left|y_{i}\right|: i \in \Omega^{h}\right\}<\infty\right\},
$$

that is, $C\left(\Omega^{\dagger}\right)$ is the $(N+1)$-dimensional Banach space of real column vectors with the maximum norm.

We will also require a discrete Volterra kernel and discrete iterated kernels.

Definition 3.2. Let $h, N$ be defined as in Definition 3.1. The discrete function $k_{i j}$ defined on the integers $i, j, 0 \leqslant j, i \leqslant N$, is said to be a discrete Volterra kernel if $k_{i j}=0$ for $j>i$. 
The discrete iterated kernels $k_{i j}^{(n)}(n=1,2, \ldots)$ of the discrete Volterra kernel $k_{i j}$ are defined to be

$$
\begin{aligned}
& k_{i j}^{(1)}=k_{i j} \\
& k_{i j}^{(n)}=h \sum_{i=j}^{i} k_{i l} k_{i j}^{(n-1)}, \quad n \geqslant 2 .
\end{aligned}
$$

It will be assumed that $0 \leqslant h k_{i i}<1$ for each $i \in \Omega^{k}$ and all $h \in I$ sufficiently small. For simplicity of notation we will then find it convenient to introduce the discrete Volterra kernel $k_{i j}$ defined as follows:

$$
k_{i j}= \begin{cases}\frac{k_{i j}}{1-h k_{i i}}, & j<i \\ 0, & j=i\end{cases}
$$

which has discrete iterated kernels $\hat{k}_{i}^{(n)}(n=1,2, \ldots)$ given by

$$
\begin{aligned}
& k_{i j}^{(1)}=k_{i j} \\
& k_{i j}^{(n)}=h \sum_{i=j+1}^{i-1} k_{i l} k_{i j}^{(n-1)}, n \geqslant 2 .
\end{aligned}
$$

We now introduce the discrete fundamental form.

Definition 3.3. The approximating problem (1.2) will be said to have a discrete fundamental form if it can be expressed in the form

$$
y^{k}=g^{k}+H^{k}\left(y^{k}\right)
$$

where $g^{k} \in C\left(\Omega^{h}\right)$ and $H^{k}: C\left(\Omega^{h}\right) \rightarrow C\left(\Omega^{h}\right)$ satisfies

$$
\begin{gathered}
\left(\left|H^{h}\left(y^{h}\right)-H^{k}\left(z^{h}\right)\right|\right)_{l} \leqslant\left(K^{k}\left|y^{h}-z^{k}\right|\right)_{l}, \\
\text { for all } i \in \Omega^{k} \text { and all } y^{h}, z^{h} \in C\left(\Omega^{h}\right),
\end{gathered}
$$

where

$$
\left|y^{k}\right|=\left(\left|y_{0}\right|,\left|y_{1}\right|, \ldots,\left|y_{N}\right|\right)^{T}, \quad\left(K^{k} y^{h}\right)_{i}=h \sum_{j=0}^{i} k_{i j} y_{f} \quad i \in \Omega^{k},
$$

and the discrete Volterra kernel $k_{l j}$ satisfies, for all $h \in I$ sufficiently small, $0 \leqslant h k_{i i}<1$ for each $i \in \Omega^{k}$ and

(DI) $k_{i j} \geqslant 0$ for $0 \leqslant j \leqslant i \leqslant N$,

(DII) for each $i \in \Omega^{h}, h \sum_{j=0}^{i-1} k_{i j}$ is bounded independently of $h$

(DII) there exists some $\mu \in \mathbf{N}$ such that the $\mu$ th discrete iterated kernel $\left.k_{i j}()^{2}\right)$ of $k_{i j}$ is bounded in $i, j$, independently of $h$.

Here $k_{i j}$ is given by (3.1).

In analogy with Theorem 2.1 we have the following existence result for the discrete fundamental form.

THEOREM 3.1 The discrete fundamental form defined by Definition 3.3 has a unique solution $y^{h} \in C\left(\Omega^{h}\right)$ for all $h \in I$ sufficiently small.

The proof follows that of Theorem 2.1 using the discrete space in place of $C[0, T]$. 
We let $f^{k}=(D)$ satisfy the discrete Volterra equation

$$
f_{i}=1+h \sum_{j=0}^{l} k_{i j} f_{p} \quad i=0,1, \ldots, N,
$$

where $k_{i j}$ is defined in definition 3.3.

It can be shown that (3.4) has a unique solution $f^{h} \in C\left(\Omega^{h}\right)$ and that $f_{i} \geqslant 1$, $0 \leqslant i \leqslant N$ (see Dixon \& McKee, 1984).

By defining a norm $\|\cdot\|_{h}$ on $C\left(\Omega^{t}\right)$ to be

$$
\left\|y^{k}\right\|_{k}=\max \left\{\frac{1}{f_{i}}\left|y_{i}\right|: i \in \Omega^{\mu}\right\},
$$

where $f^{h}$ is the solution of (3.4), and then proceeding as in the proof of Theorem 2.1 we deduce that there exists a unique $y^{h} \in C\left(\Omega^{k}\right)$ satisfying

$$
y^{h}=\theta^{k}+H^{h}\left(y^{h}\right) \text {. }
$$

The following theorem, which may be deduced from a result given by Dixon \& McKee (1984), is the discrete analogue of Theorem 2.2.

THEOREM 3.2 Let $h \in I$ be sufficiently small.

For any $\phi^{k} \in C\left(\Omega^{k}\right)$ if $x^{k} \in C\left(\Omega^{k}\right)$ satisfies

$$
x_{i} \leqslant \phi_{i}+h \sum_{j=0}^{i} k_{i j} x_{j} \quad i=0,1, \ldots, N,
$$

where the discrete Volterra kernel $k_{i j}$ satisfies (DI)-(DII), then

$$
x_{i} \leqslant y_{i}, \quad i=0,1, \ldots, N,
$$

where $y^{h}$ is the unique $C\left(\Omega^{h}\right)$ solution of the discrete integral equation

$$
y_{i}=\phi_{i}+h \sum_{j=0}^{i} k_{i j} y_{j}, i=0,1, \ldots, N .
$$

Moreover, there exists a constant $C$, independent of $\phi^{h}$ and $h$, such that if $x^{k} \geqslant 0$ and $\phi^{h} \geqslant 0$

$$
\left\|x^{k}\right\|_{\infty} \leqslant C\left\|\phi^{h}\right\|_{\infty}
$$

\section{Prolongation and Restriction Operators}

The problem of error estimates is that of comparing $y^{h} \in X^{h}$ with $y \in X$ objects in different spaces. We overcome this difficulty by introducing prolongation and restriction operators, which are simply linear operators from $X^{k}$ into $X$, and $X$ into $X^{h}$ respectively, which will be required to satisfy certain conditions. This enables us to reduce the problem of comparing $y^{k}$ and $y$ to that of considering the effect of perturbations in the fundamental forms (21) and (3.2).

Consider the operator equation

$$
y=g+H(y), \quad H: X \rightarrow X .
$$

and the corresponding discrete equation

$$
y^{k}=g^{k}+H^{k}\left(y^{k}\right), \quad H^{k}: X^{h} \rightarrow X^{k} .
$$


For general Banach spaces $X, X^{h}$ with $h \in I$, inf $I=0$, we will require the prolongation operator $p^{h}$ and the restriction operator $r^{h}$ to have the following properties:

(a) $r^{k}: X \rightarrow X^{h}$ is a bounded linear operator with

$$
\left\|r^{k}\right\| \leqslant 1 \text { for all } h \in I \text {. }
$$

(b) $p^{h}: X^{h} \rightarrow X$ is a bounded linear operator, and there exists $p$, independent of $h$, such that

$$
\left\|p^{n}\right\| \leqslant p
$$

(c) $r^{k} p^{h}=I^{k}$, the identity operator on $X^{h}$.

(d) For all $x \in X$ sufficiently smooth

$$
\left\|\left(1-p^{h} r^{h}\right) x\right\|_{X} \rightarrow 0 \text { as } h \rightarrow 0 .
$$

It is important to consider $x$ fixed as $h \rightarrow 0$ since

$$
\left\|1-p^{k} r^{k}\right\|+0 \text { as } h \rightarrow 0 \text { in general. }
$$

(e) For all $x^{k} \in X^{k}$ with $\left\|x^{k}\right\|_{x^{n}}$ uniformly bounded for all $h \in I$

$$
\left\|\left(1-p^{k} r^{k}\right) H\left(p^{k} x^{k}\right)\right\|_{x} \rightarrow 0 \text { as } h \rightarrow 0 .
$$

(f) For all $x^{h} \in X^{h}$ with $\left\|x^{k}\right\|_{x^{n}}$ uniformly bounded for all $h \in I$,

$$
\left\|r^{h} H\left(p^{k} x^{h}\right)-H^{h}\left(x^{h}\right)\right\|_{x^{h}} \rightarrow 0 \text { as } h \rightarrow 0 .
$$

In the case $H(y)=H y$ and $H^{k}\left(y^{h}\right)=H^{k} y^{h}$, that is, $H$ and $H^{k}$ are linear operators, the conditions (e) and (f) will be replaced by $\left(\mathrm{e}^{1}\right)$ and $\left(\mathrm{f}^{1}\right)$ as follows:

$$
\left\|\left(1-p^{k} r^{k}\right) H\right\| \rightarrow 0 \text { as } h \rightarrow 0 .
$$

This will be automatically satisfied if $H$ is a compact operator.

$$
\left\|r^{k} H p^{h}-H^{h}\right\| \rightarrow 0 \text { as } h \rightarrow 0 .
$$

The framework we have set up is general, but the restriction and prolongation operators must be tailored to the particular discretization method under consideration. It should be arranged that $p^{k}, r^{h}$ are chosen so that

$$
\left\|\left(1-p^{k} r^{k}\right) H\left(p^{k} x^{h}\right)\right\|_{x} \text { and }\left\|r^{h} H\left(p^{h} x^{h}\right)-H^{k}\left(x^{h}\right)\right\|_{x^{h}}
$$

are small and of the same order in $h$. It is also desirable that $\left\|p^{k}\right\|$ [which is $\geqslant 1$ by assumptions (a) and (c)] be as near one as possible, since it will occur as a multiplying factor in our error bounds.

We require estimates of $\left\|p^{k} y^{h}-y\right\|_{x}$ and $\left\|r^{k} y-y^{k}\right\|_{x^{n}}$. It will be assumed in the remainder of this section that $X=C[0, T]$ and $X^{h}=C\left(\Omega^{h}\right)$, the required error estimates then being obtained by applying the generalized continuous and discrete Gronwall inequalities (Theorems 2.2 and 3.2) respectively. 
THEOREM 4.1 Let $y$ satisfy the analytic fundamental form (2.1) and let $y^{h}$ satisfy the discrete non-linear operator equation

where $H^{h}: C\left(\Omega^{h)} \rightarrow C\left(\Omega^{h}\right)\right.$.

$$
y^{h}=g^{k}+H^{k}\left(y^{h}\right)
$$

Let $p^{k}, r^{h}$ be respectively a prolongation and restriction operator with the properties (a) $-($ ()).

Then if $g^{k}=r^{h} g$ there exists a constant $C_{p}$ independent of $h$, such that

$$
\begin{aligned}
& \left\|p^{h} y^{h}-y\right\|_{\infty} \leqslant C_{p}\left\{p\left\|H^{h}\left(y^{h}\right)-r^{h} H\left(p^{h} y^{h}\right)\right\|_{\infty}+\right. \\
& \left.\left\|\left(1-p^{k} r^{k}\right) H\left(p^{k} y^{k}\right)\right\|_{\infty}+\left\|\left(1-p^{k} r^{k}\right) g\right\|_{\infty}\right\}
\end{aligned}
$$

and $p^{k} y^{h} p$-converges to $y$.

Proof. Applying the prolongation operator $p^{h}$ to the discrete equation (4.1) with $g^{k}=r^{k} g$ yields

$$
p^{h} y^{h}=p^{h} H^{h}\left(y^{h}\right)+p^{h} r^{h} g .
$$

Therefore, $p^{k} y^{k} \in C[0, T]$ satisfies the perturbed analytic fundamental form

where

$$
p^{k} y^{k}=H\left(p^{k} y^{k}\right)+g+\theta
$$

$$
\theta=\left(p^{h} H^{k}\left(y^{h}\right)-H\left(p^{k} y^{h}\right)\right)+\left(p^{k} \mu^{k}-1\right) g \in C[0, T] .
$$

Subtracting the fundamental form (2.1), $p^{k} y^{k}-y$ satisfies

$$
p^{k} y^{k}-y=H\left(p^{k} y^{h}\right)-H(y)+\theta \text {. }
$$

Using condition (2.2),

$$
\left|p^{k} y^{k}-y\right| \leqslant K\left|p^{k} y^{k}-y\right|+|\theta| \text {. }
$$

Invoking Theorem 22 with $x=\left|p^{h} y^{h}-y\right| \geqslant 0$ we deduce that there exists some constant $C_{p}$ such that

Recall that

$$
\left\|p^{h} y^{h}-y\right\|_{\infty} \leqslant C_{p}\|\theta\|_{\infty}
$$

$$
\begin{aligned}
\theta & =\left(p^{k} H^{k}\left(y^{h}\right)-H\left(p^{k} y^{h}\right)\right)+\left(p^{k} r^{h}-1\right) g \\
& =p^{k}\left(H^{k}\left(y^{k}\right)-r^{k} H\left(p^{k} y^{k}\right)\right)+\left(p^{k} r^{k}-1\right) H\left(p^{k} y^{k}\right)+\left(p^{h} r^{k}-1\right) g .
\end{aligned}
$$

Hence

$$
\|\theta\|_{\infty} \leqslant p\left\|H^{h}\left(y^{k}\right)-r^{h} H\left(p^{k} y^{k}\right)\right\|_{\infty}+\left\|\left(1-p^{h} r^{h}\right) H\left(p^{k} y^{h}\right)\right\|_{\infty}+\left\|\left(1-p^{k} r^{h}\right) g\right\|_{\infty}
$$

and the bound (4.2) follows.

Letting $h \rightarrow 0$ we deduce using (d), (e), (f) that $\left\|p^{k} y^{h}-y\right\|_{\infty} \rightarrow 0$.

We note that the bound on $\|\theta\|_{\infty}$ is computable since it does not depend on the unknown function $y$. This implies that the bound for $\left\|p^{k} y^{k}-y\right\|_{\infty}$ is computable if the constant $C_{p}$ can be found explicitly.

We also remark that if $g^{b} \neq r^{h} g$ then the additional condition $\left\|g^{h}-r^{k} g\right\|_{\omega} \rightarrow 0$ as $h \rightarrow 0$ is required.

THEOREM 4.2 Let $y, y^{k}$ satisfy the fundamental forms (2.1) and (3.2) respectively and let $p^{k}, r^{k}$ be respectively a prolongation and restriction operator with the properties (a), (d), (f). 
Then for $h \in I$ sufficiently small if $g^{h}=r^{h} g$ there exists a constant $C_{r}$, independent of $h$, such that

$$
\left\|r^{k} y-y^{k}\right\|_{\infty} \leqslant C_{r}\left\{\left\|H^{k}\left(r^{k} y\right)-r^{k} H\left(p^{k} r^{k} y\right)\right\|_{\infty}+\|K\|\left\|\left(1-p^{k} r^{k}\right) y\right\|_{\infty}\right\}
$$

and $y^{h}$ r-converges to $y$.

Proof. Premultiplying the fundamental form (2.1) by $r^{k}$,

$$
r^{h} y=r^{h} H(y)+r^{h} g \text {. }
$$

Therefore, since by assumption $g^{h}=r^{h} g, r^{h} y$ satisfies the perturbed discrete fundamental form

$$
r^{k} y=H^{h}\left(r^{k} y\right)+g^{k}+\theta^{k},
$$

where $\theta^{h}=r^{h} H(y)-H^{k}\left(r^{k} y\right) \in C\left(\Omega^{k}\right)$ is the consistency error. Subtracting the discrete fundamental form (3.2), $r^{h} y-y^{h}$ satisfies

$$
r^{h} y-y^{h}=H^{h}\left(r^{h} y\right)-H^{h}\left(y^{h}\right)+\theta^{h}
$$

Using condition (3.3),

$$
\left|r^{k} y-y^{k}\right| \leqslant K^{h}\left|r^{k} y-y^{h}\right|+\left|\theta^{h}\right| \text {. }
$$

An application of Theorem 3.2 with $x^{h}=\left|r^{h} y-y^{h}\right| \geqslant 0$ allows us to conclude that for $h$ sufficiently small there exists $C_{r}$, independent of $h$, such that

But

$$
\left\|r^{h} y-y^{h}\right\|_{\infty} \leqslant C_{r}\left\|\theta^{h}\right\|_{\infty} \text {. }
$$

$$
\begin{aligned}
\theta^{k} & =r^{k} H(y)-H^{k}\left(r^{k} y\right) \\
& =\left(r^{k} H\left(p^{k} r^{k} y\right)-H^{k}\left(r^{k} y\right)\right)+r^{k}\left(H(y)-H\left(p^{h} r^{h} y\right)\right) .
\end{aligned}
$$

Therefore, using the Lipschitz condition (2.2),

$$
\left|\theta^{k}\right| \leqslant\left|r^{k} H\left(p^{k} r^{k} y\right)-H^{k}\left(r^{k} y\right)\right|+\left|r^{k}\right| K\left|y-p^{k} r^{k} y\right|
$$

and taking norms and using condition (a),

$$
\left\|\theta^{k}\right\|_{\infty} \leqslant\left\|r^{k} H\left(p^{k} r^{k} y\right)-H^{k}\left(r^{k} y\right)\right\|_{\infty}+\|K\|\left\|\left(1-p^{k} r^{k}\right) y\right\|_{\infty},
$$

from which (4.3) follows.

We deduce, using (d) and (f), that since $\|K\|$ is bounded, $\left\|r^{k} y-y^{k}\right\|_{\infty} \rightarrow 0$ as $h \rightarrow 0$.

We observe that the estimate (4.3) for $\left\|r^{h} y-y^{k}\right\|_{\infty}$ suffers from the disadvantage of not being directly computable since it depends on the unknown function $y$. However, if $y$ has a prescribed degree of smoothness, (4.3) may be used to determine the rate of convergence.

We note that if $r^{k} p^{k}=I^{h}$, then

$$
\left\|r^{h} y-y^{h}\right\|_{\infty}=\left\|r^{k}\left(y-p^{k} y^{h}\right)\right\|_{\infty} \leqslant\left\|y-p^{h} y^{k}\right\|_{\infty}
$$

and Theorem 4.1 is applicable provided (a)-(f) are satisfied. We conclude that a bound on $\left\|r^{h} y-y^{k}\right\|_{\infty}$ which is at least as good as that on $\left\|y-p^{h} y^{h}\right\|_{\infty}$ is then possible. 


\section{A Gronwall Inequality for Abstract Operators}

We wish to extend our results from $X=C[0, T]$ (and $X^{h}=C\left(\Omega^{h}\right)$ ) to more general Banach spaces. To do this we must first generalize our Gronwall inequalities.

The following lemma is a general fixed point result.

LeMma 5.1 Let $X$ be a Banach space and let $S \in L(X)$ (the space of bounded linear operators from $X$ into itself) be such that for some $n \in \mathbf{N} S^{n}$ is a contraction. For each $z \in X$ the operator $T_{x}$ defined by $T_{x} x=z+S x$ has a unique fixed point $x \in X$ given by

$$
x=\sum_{r=0}^{\infty} S_{z}
$$
Proof. Since $S^{n}$ is a contraction for some $n$, it can be shown that $\sum_{r=0}^{\infty} S^{r}$ converges in
$L(X)$ and, moreover

$$
(I-S)^{-1}=I+S+S^{2}+\ldots
$$

Thus $x=z+S x$ has a unique solution given by

$$
x=(I-S)^{-1} z=\sum_{r=0}^{\infty} S^{r} z \in X .
$$

(See also Hille, 1969, 1972.)

Following the argument used to obtain the Gronwall inequality given by Theorem 2.2 in the space $X=C[0, T]$ (see Dixon \& McKee, 1984) we introduce, for any Banach space $X$, a linear operator $T: X \rightarrow X$ defined by

$$
T x=x-S x \text {, }
$$

where $S \in L(X)$ and $S^{n}$ is a contraction for some $n$.

LEMMA 5.2 Let $X$ be a partially ordered Banach space whose positive cone $X^{+}:=\{x \in X: x \geqslant 0\}$ is closed. If $S \in L(X)$ is a positive operator (that is, $S X^{+} \subseteq X^{+}$) such that for some $n \in \mathbf{N} S^{n}$ is a contraction, then $T x \in X^{+}$implies $x \in X^{+}$.

Proof. If $T x=z \in X^{+}$, then $x-S x=z$.

By Lemma 5.1

$$
x=\sum_{r=0}^{\infty} S_{z}
$$

Since $z \in X^{+}$and $S$ is a positive operator, $S z \in X^{+}$and by induction $S^{+} z \in X^{+}$for all $r \geqslant 0$.

Consequently,

$$
\sum_{r=0}^{m} S^{r} z \in X^{+} \text {for all } m \geqslant 0
$$

But $X^{+}$is closed and hence $x \in X^{+}$.

The following Gronwall inequality is an immediate consequence of Lemma 5.2 (see also Beesack, 1975).

THEOREM 5.1 Let $X$ be a partially ordered Banach space whose positive cone $X^{+}$is closed, and let $S \in L(X)$ be a positive operator such that for some $n \in \mathbf{N} S^{n}$ is a contraction. 
For any $z \in X$ if $x \in X$ satisfies the inequality

then

$$
\begin{gathered}
x \leqslant z+S x \\
x \leqslant y,
\end{gathered}
$$

where $y$ is the unique solution in $X$ of the equation

$$
y=z+S y .
$$

Proof. Using the definition of the operator $T$,

$$
\begin{aligned}
T(y-x) & =(y-x)-S(y-x) \\
& =(y-S y)-(x-S x) \geqslant 0 .
\end{aligned}
$$

Therefore, $T(y-x) \in X^{+}$and Lemma 5.2 implies $y-x \in X^{+}$.

Consequently,

$$
x \leqslant y \text {. }
$$

Corollary 5.1. If, in addition to the conditions stated in Theorem 5.1, $X$ is a Banach lattice then there exists a constant $C$, independent of $z$, such that if $x \in X^{+}$and $z \in X^{+}$

$$
\|x\| \leqslant C\|z\|,
$$

where $\|\cdot\|$ denotes the norm in $X$.

Proof. By (5.4) $x \leqslant y$ and since $x \in X^{+}$

$$
|x| \leqslant|y| \text {. }
$$

Hence by the compatibility of the norm and order relation

By Lemma 5.1

$$
\|x\| \leqslant\|y\| \text {. }
$$

and hence

$$
y=\sum_{r=0}^{\infty} S^{r} z
$$

$$
\|y\| \leqslant \sum_{r=0}^{\infty}\left\|S^{r}\right\|\|z\|
$$

Now $S^{n}$ is a contraction and therefore $\sum^{\infty}=0\left\|S^{r}\right\|$ converges and we deduce that there exists some $C$, independent of $z$, such that

$$
\|x\| \leqslant C\|z\| \text {. }
$$

\section{Prolongation and Restriction Operators in Banach Spaces}

Consider again the non-linear operator equation

$$
\Phi(y)=0, \quad \Phi: X \rightarrow Y,
$$

and the corresponding discrete operator equation

$$
\Phi^{h}\left(y^{h}\right)=0, \quad \Phi^{k}: X^{h} \rightarrow Y^{k} .
$$

In the subsequent discussion it will be assumed that $X, X^{h}$ are both Banach lattices, and $|\cdot|$ will denote the absolute value in $X$ and $X^{h}$. We also assume that (6.1) and (6.2) have unique solutions $y \in X$ and $y^{k} \in X^{k}$ respectively. 
Definition 6.1. Equation (6.1) will be said to have an analytic fundamental form if it can be expressed in the form

$$
y=g+H(y)
$$

where $g \in X$ and $H: X \rightarrow X$ satisfies

$$
|H(y)-H(z)| \leqslant K|y-z|, \text { for all } y, z \in X,
$$

where $K \in L(X)$ is a positive operator such that $K^{n}$ is a contraction for some $n$.

Analogically we have the following definition of a discrete fundamental form.

Definition 6.2. Let $h_{0}>0$ and let $I$ be a non-empty subset of $\left(0, h_{0}\right]$ with inf $I=0$. Equation (6.2) will be said to have a discrete fundamental form if for each $h \in I$ it can be expressed in the form

$$
y^{k}=g^{h}+H^{k}\left(y^{h}\right)
$$

where $g^{h} \in X^{h}$ and $H^{h}: X^{h} \rightarrow X^{h}$ satisfies

$$
\left|H^{k}\left(y^{h}\right)-H^{h}\left(z^{h}\right)\right| \leqslant K^{h}\left|y^{h}-z^{h}\right|, \quad \text { for all } y^{h}, z^{h} \in X^{k},
$$

where $K^{h} \in L\left(X^{h}\right)$ is a positive operator bounded independently of $h$, such that $\left(K^{h}\right)^{n}$ is a contraction for some $n \in \mathbf{N}$ with $\left\|\left(K^{h}\right)^{\mu}\right\| \leqslant C<1$, where $C$ is independent of $h$.

We now present generalizations of Theorems 4.1 and 4.2. As in Section 4, $p^{h}, r^{k}$ will denote respectively a prolongation and restriction operator with the properties (a)-(f), where $X, X^{h}$ are now Banach lattices.

TheOReM 6.1 Let $X, X^{h}$ be Banach lattices with $X^{+}$closed. Let $y$ satisfy (6.3) and $y^{h}$ satisfy the discrete operator equation

$$
y^{h}=g^{k}+H^{k}\left(y^{h}\right), \quad H^{k}: X^{h} \rightarrow X^{k} .
$$

Then if $p^{h}, r^{h}$ have properties (a)-(f) and $g^{h}=r^{h} g$, there exists a constant $C_{p}$, independent of $h$, such that

$$
\left\|p^{h} y^{h}-y\right\|_{x} \leqslant C_{p}\left\{p\left\|H^{k}\left(y^{h}\right)-r^{h} H\left(p^{k} y^{h}\right)\right\|_{x^{h}+}\left\|\left(1-p^{k} r^{h}\right) H\left(p^{h} y^{h}\right)\right\|_{x}+\left\|\left(1-p^{k} r^{h}\right) g\right\|_{x}\right\}
$$

and $p^{k} y^{k} p$-converges to $y$.

Proof. The difference $p^{k} y^{k}-y$ satisfies

where

$$
p^{k} y^{k}-y=H\left(p^{k} y^{k}\right)-H(y)+\theta,
$$

$$
\theta=\left(p^{k} H^{k}\left(y^{k}\right)-H\left(p^{k} y^{k}\right)\right)+\left(p^{k} r^{k}-1\right) g \in X .
$$

Since $X$ is a Banach lattice

$$
\begin{aligned}
\left|p^{k} y^{h}-y\right| & \leqslant\left|H\left(p^{k} y^{k}\right)-H(y)\right|+|\theta| \\
& \leqslant K\left|p^{k} y^{k}-y\right|+|\theta|
\end{aligned}
$$

using the Lipschitz condition (6.4).

Invoking Corollary 5.1 with

$$
x=\left|p^{h} y^{h}-y\right| \in X^{+}, \quad z=|\theta| \in X^{+},
$$


we deduce that there exists $C_{p}$ such that

$$
\left\|p^{k} y^{k}-y\right\|_{x} \leqslant C_{p}\|\theta\|_{x} .
$$

The required bound (6.8) follows exactly as in the proof of Theorem 4.1.

THEOREM 6.2 Let $X, X^{h}$ be Banach lattices with $\left(X^{h}\right)^{+}$closed, and let $y, y^{h}$ satisfy the fundamental forms (6.3) and (6.5) respectively.

Then if $p^{k}, r^{k}$ have properties $(a),(d),(f)$ and $g^{k}=r^{k} g$, there exists a constant $C_{r}$, independent of $h$, such that for $h \in I$ sufficiently small

$$
\left\|r^{h} y-y^{h}\right\|_{x^{h}} \leqslant C_{r}\left\{\left\|H^{h}\left(r^{k} y\right)-r^{k} H\left(p^{k} r^{h} y\right)\right\|_{x^{n}}+\|K\|\left\|\left(1-p^{h} r^{h}\right) y\right\|_{x}\right\}
$$

and $y^{h}$ r-converges to $y$.

Proof. The difference $r^{k} y-y^{h}$ satisfies

where

$$
\begin{aligned}
r^{k} y-y^{k} & =H^{k}\left(r^{k} y\right)-H^{k}\left(y^{k}\right)+\theta^{k}, \\
\theta^{k} & =r^{k} H(y)-H^{k}\left(r^{k} y\right) .
\end{aligned}
$$

Therefore, since $X^{h}$ is a Banach lattice and $H^{k}$ satisfies (6.6),

$$
\left|r^{h} y-y^{h}\right| \leqslant K^{h}\left|r^{k} y-y^{k}\right|+\left|\theta^{h}\right| \text {. }
$$

Applying Corollary 5.1 with

$$
x=\left|r^{k} y-y^{h}\right| \in\left(X^{h}\right)^{+}, \quad z=\left|\theta^{k}\right| \in\left(X^{h}\right)^{+},
$$

we deduce that there exists $C_{r}$ such that

$$
\left\|r^{k} y-y^{k}\right\|_{x^{h}} \leqslant C_{r}\left\|\theta^{k}\right\|_{x^{h}}
$$

To show $C_{r}$ is independent of $h$ we must demonstrate that $\sum_{r=0}^{\infty}\left\|\left(K^{h}\right)\right\|$ is bounded independently of $h$. Any $r \in \mathbf{N}$ may be expressed uniquely in the form

ana

$$
r=n m+q \text {, for some } m \geqslant 0,0 \leqslant q \leqslant n-1 \text {, }
$$

$$
\sum_{r=0}^{\infty}\left\|\left(K^{h}\right)^{r}\right\| \leqslant \sum_{m=0}^{\infty}\left\|\left(K^{h}\right)^{m}\right\|^{m} \sum_{q=0}^{n-1}\left\|\left(K^{h}\right)^{q}\right\| \text {. }
$$

By hypothesis there exists $M$, independent of $h$, such that $\left\|K^{h}\right\|<M$ and consequently $\left\|\left(K^{n}\right)^{q}\right\|<M^{q}, 0 \leqslant q \leqslant n-1$.

Also there exists $C$, independent of $h$, such that $\left\|\left(K^{h}\right)^{x}\right\| \leqslant C<1$ and therefore

We deduce that

$$
\sum_{m=0}^{\infty}\left\|\left(K^{h}\right)^{m}\right\|^{m} \leqslant \frac{1}{1-C}
$$

$$
\sum_{r=0}^{\infty}\left\|\left(K^{k}\right)\right\| \leqslant C_{r}
$$

where $C_{r}$ is independent of $h$.

It remains to consider $\left\|\theta^{\prime}\right\|_{\text {m }}$.

Recall that

$$
\begin{aligned}
\theta^{h} & =r^{h} H(y)-H^{k}\left(r^{h} y\right) \\
& =\left(r^{h} H\left(p^{h} r^{h} y\right)-H^{k}\left(r^{h} y\right)\right)+r^{h}\left(H(y)-H\left(p^{h} r^{h} y\right)\right) .
\end{aligned}
$$


Using (6.4)

$$
\left|H(y)-H\left(p^{k} r^{h} y\right)\right| \leqslant K\left|y-p^{h} r^{k} y\right|,
$$

where $K$ is a positive operator.

Hence by the compatibility of the norm and partial ordering

$$
\left\|H(y)-H\left(p^{h} r^{h} y\right)\right\|_{x} \leqslant\|K\|\left\|\left(1-p^{h} \tau^{h}\right) y\right\|_{x} .
$$

Consequently,

$$
\left\|\theta^{k}\right\|_{X^{h}} \leqslant\left\|H^{k}\left(r^{k} y\right)-r^{h} H\left(p^{h} r^{h} y\right)\right\|_{X^{h}}+\|K\|\left\|\left(1-p^{h} r^{h}\right) y\right\|_{X},
$$

and the required bound (6.9) follows.

\section{An Example}

We illustrate the above theory by studying the convergence of a collocation method for second-kind Volterra integral equations.

Consider the equation

$$
y(t)=g(t)+\int_{0}^{t} \frac{G(t, s, y(s))}{(t-s)^{\alpha}} d s, \quad 0 \leqslant t \leqslant T,
$$

where $0 \leqslant \alpha<1$, and $g, G$ are assumed to satisfy suitable continuity conditions.

Given a positive integer $N$, let $t_{t}=i h, 0 \leqslant i \leqslant N$, where $h=T / N$, and set $\sigma_{t}=\left(t_{b} t_{t+1}\right], 1 \leqslant i \leqslant N-1$, with $\sigma_{0}=\left[t_{0}, t_{1}\right]$.

Given $m \geqslant 1$ and collocation parameters

define

$$
0<\eta_{1}<\eta_{2}<\ldots<\eta_{m} \leqslant 1
$$

$$
Q_{N}:=\left\{t=t_{i v}: t_{i v}=t_{i}+\eta_{v} h_{v}, 1 \leqslant v \leqslant m, 0 \leqslant i \leqslant N-1\right\} .
$$

Let

$$
S(m-1, N):=\left\{u:\left.u\right|_{\sigma_{1}} \in \Pi_{m-1}, 0 \leqslant i \leqslant N-1\right\}
$$

denote the space of piecewise polynomials of degree $m-1$ which possess (possibly) finite (jump) discontinuities at the points $t_{i}, 1 \leqslant i \leqslant N-1$.

The collocation method of approximating (7.1) is then given by

$$
u(t)=g(t)+\int_{0}^{t} \frac{G(t, s, u(s))}{(t-s)^{\alpha}} d s, \quad t \in Q_{N}, \quad u \in S(m-1, N) .
$$

(See, for example, Brunner, 1978.)

It is now necessary to formulate the integral equation (7.1) and the corresponding collocation (7.2) in fundamental form and to construct appropriate prolongation and restriction operators.

Let $X=B[0, T]$, the space of bounded functions on $[0, T]$ which are continuous almost everywhere, with the supremum norm

$$
\|x\|_{\infty}=\sup \{|x(t)|: 0 \leqslant t \leqslant T\},
$$


and for all $x, y \in X$ define the order relation

$$
x \leqslant y \text { if } x(t) \leqslant y(t) \text { for all } t \in[0, T] .
$$

Then $|x(t)| \leqslant|y(t)|$ for all $t \in[0, T]$ implies $\|x\|_{\infty} \leqslant\|y\|_{\infty}$, so that the norm is compatible with the order relation.

In operator notation the integral equation (7.1) may be written as

$$
y=g+H(y)
$$

where $g \in X$ and $H: X \rightarrow X$ satisfies

$$
|H(y)-H(z)| \leqslant K|y-z|, \text { for all } y, z \in X,
$$

with

$$
K y(t)=L \int_{0}^{t} \frac{y(s)}{(t-s)^{\alpha}} d s, \quad 0 \leqslant t \leqslant T .
$$

Here $L$ is the Lipschitz constant of $G(t, s, y)$ with respect to $y$. It is straightforward to show that since $0 \leqslant \alpha<1,\left\|K^{\prime \prime}\right\|<1$ for all $n$ sufficiently large. Consequently, (7.3) is the analytic fundamental form for (7.1).

Let $X^{h}$ be the Banach space of real column vectors $x^{h}=\left(x_{i v}\right), x_{i v} \in \mathbf{R}, 1 \leqslant v \leqslant m$, $0 \leqslant i \leqslant N-1$, with norm

$$
\left\|x^{h}\right\|_{\infty}=\max \left\{\left|x_{i v}\right|: 1 \leqslant v \leqslant m, 0 \leqslant i \leqslant N-1\right\},
$$

and for all $x^{h}, y^{h} \in X^{h}$ define the order relation

$$
x^{h} \leqslant y^{h} \quad \text { if } x_{i v} \leqslant y_{i v} \text { for all } i, v, \quad 1 \leqslant v \leqslant m, \quad 0 \leqslant i \leqslant N-1 .
$$

Define a restriction operator $r^{h}: X \rightarrow X^{h}$ by

$$
\left(r^{h} x(t)\right)_{i v}=x\left(t_{i v}\right), \quad 1 \leqslant v \leqslant m, \quad 0 \leqslant i \leqslant N-1,
$$

and a prolongation operator $p^{h}: X^{h} \rightarrow X$ by

$$
\left(p^{h} x^{h}\right)(t)=\sum_{v=1}^{m} x_{i v} L_{v}\left(\frac{t-t_{i}}{h}\right), \quad t \in \sigma_{i}, \quad 0 \leqslant i \leqslant N-1,
$$

where

$$
L_{v}(t)=\prod_{t \rightarrow v}\left(\frac{t-\eta_{t}}{\eta_{v}-\eta_{t}}\right), \quad 1 \leqslant v \leqslant m
$$

are the Lagrange fundamental polynomials for the points $\eta_{1}, \eta_{2}, \ldots, \eta_{m}$

The approximation $u \in S(m-1, N) \subset X$ to $y$ obtained by collocation is a piecewise polynomial of degree $m-1$ and consequently may be represented as

$$
u(t)=\sum_{i=1}^{m} u\left(t_{i v}\right) L_{r}\left(\frac{t-t_{i}}{h}\right), \quad t \in \sigma_{i}, \quad 0 \leqslant i \leqslant N-1 .
$$

Hence using (7.4) the collocation (7.2) may be rewritten in the form

$$
r^{h} u=g^{k}+H^{h}\left(r^{h} u\right),
$$


where $g^{k}=r^{h} g \in X^{h}$ and $H^{k}: X^{h} \rightarrow X^{k}$ is given by

$$
\begin{aligned}
\left(H^{k}\left(x^{k}\right)\right)_{i v}= & \sum_{j=0}^{i-1} \int_{t_{j}}^{t_{j+1}} \frac{G\left(t_{i v} s, \sum_{i=1}^{m} x_{h} L_{i}\left(\frac{s-t_{j}}{h}\right)\right)}{\left(t_{i v}-s\right)^{a}} d s+ \\
& \quad \int_{t_{i}}^{t_{i v}} \frac{G\left(t_{i v} s, \sum_{i=1}^{m} x_{i \tau} L_{i}\left(\frac{s-t_{i}}{h}\right)\right)}{\left(t_{i v}-s\right)^{x}} d s, \quad 1 \leqslant v \leqslant m, \quad 0 \leqslant i \leqslant N-1 .
\end{aligned}
$$

We must now check the properties of $p^{h}, r^{h}$.

(a) $r^{h}: X \rightarrow X^{k}$ is a bounded linear operator with $\left\|r^{k}\right\|=1$.

(b) $p^{h}: X^{h} \rightarrow X$ is a bounded linear operator and there exists $p_{m}$ (depending on $m$ but not on $h$ ) such that $\left\|p^{h}\right\| \leqslant p_{m}$.

(c) $r^{k} p^{k}=I^{k}$.

(d) From Theorem 6.1 for $p$-convergence it is necessary to consider in particular $\left\|\left(1-p^{k} r^{k}\right) g\right\|_{\infty}$. The theory of Lagrange interpolation implies that if $g \in C^{m}[0, T]$, then

$$
\left(1-p^{k} r^{k}\right) g(t)=\frac{h^{m}}{m !} \prod_{n=1}^{m}\left(\frac{t-t_{l 0}}{h}\right) g^{(m)}\left(\xi_{i}\right), \quad t \in \sigma_{l},
$$

for some $\xi_{i} \in \sigma_{i}$, and consequently there exists $C_{1}$, independent of $h$, such that

$$
\left\|\left(1-p^{k} r^{k}\right) g\right\|_{\infty} \leqslant C_{1} h^{m} \text {. }
$$

(e) We must consider $\left\|\left(1-p^{k} h^{k}\right) H\left(p^{k} y^{k}\right)\right\|_{\infty}$, where in particular, $p^{k} y^{k}=p^{k} r^{k} u=u$ [by (7.4), (7.5) and (7.6)]. Again using the error in Lagrange interpolation we may deduce that, provided $G(t, s, u)$ satisfies suitable continuity conditions,

$$
\left\|\left(1-p^{k} r^{h}\right) H(u)\right\|_{\infty} \leqslant C_{2} h^{m}
$$

for some $C_{2}$ independent of $h$.

(f) Finally, it is straightforward to demonstrate that

$$
H^{k}\left(y^{k}\right)-r^{k} H\left(p^{k} y^{k}\right)=0 .
$$

Invoking Theorem 6.1 with $y^{k}=r^{k} u$ and noting that $p^{k} y^{k}=u$ we conclude that there exists $C$, independent of $h$, such that

$$
\|y-u\|_{\infty} \leqslant C h^{m} \text {. }
$$

In most practical applications the integrals (7.7) cannot be found analytically and appropriate quadrature formulae must be employed.

Approximating $H^{h}: X^{h} \rightarrow X^{h}$ by $A^{h}: X^{h} \rightarrow X^{h}$ yields an approximation $\tilde{y}^{h} \in X^{h}$ satisfying

$$
\tilde{y}^{h}=g^{h}+H^{h}\left(\tilde{y}^{h}\right)
$$

Therefore, $p^{k} \tilde{y}^{h}=\tilde{u}$ satisfies the perturbed analytic fundamental form

$$
\tilde{u}=g+H(\tilde{u})+\sigma,
$$


where

$$
\theta=\theta+p^{k}\left(\tilde{H}^{k}\left(\tilde{y}^{h}\right)-H^{k}\left(\tilde{y}^{h}\right)\right)
$$

and $\theta$ is defined as in Theorem 6.1 with $\tilde{y}^{h}$ in place of $y^{k}$. We deduce that

$$
\|y-\tilde{u}\|_{\infty} \leqslant C h^{m} \text {, }
$$

provided $H^{4}$ is chosen such that

$$
\left\|H^{h}\left(\tilde{y}^{h}\right)-H^{h}\left(\tilde{y}^{h}\right)\right\|_{\infty} \leqslant C h^{m} .
$$

Both authors are funded by the Science and Engineering Research Council and are grateful for their financial support.

\section{REFERENCES}

AUbIN, J. P. 1977 Approximations of Elliptic Boundary Vatue Problems. Wiley-Interscience. BeEsuCK, P. 1975 Gronwall Inequalities. Carleton Mathematical Lecture Notes, no. 11.

BRUNNER, H. 1978 The approximate solution of integral equations by projection methods based on collocation (lecture notes). Mathematics and Computation No. 1/78, Dept. of Math, Univ. of Trondheim.

CEA, J. 1964 Approximation variationelle des problèmes aux limites. Ann. Inst. Fourier 14, $345-441$.

DixoN, J. A. \& MCKEE, S. 1984 Weakly singular discrete integral inequalities and their application. In Proceedings of the Tenth South African Symposium on Numerical Mathematics (Snyman, J. A., Ed.).

HILLe, E. 1969 Lectures on Ordinary Differential Equations. Reading, Mass. Addison-Wesley.

HnLe, E. 1972 Methods in Classical and Functional Analysis. Reading, Mass. Addison-Wesley.

NoBLE, B. 1973 Error analysis of collocation methods for solving Fredholm integral equations. In Topics in Numerical Analysis (Miller, J. H. H., Ed.), pp. 211-232. London: Academic Press.

Thомнs, K. S. 1973 The numerical solution of integral equations. D.Phil. Thesis, Univ. of Oxford.

WRIGHT, K. 1982 Asymptotic properties of matrices associated with the quadrature method for integral equations. In Treatment of Integral Equations by Numerical Methods (Baker, C. T. H. \& Miller, G. F., Eds), pp. 325-336. London: Academic Press. 